\section{High-Performance Payload Data Handling System for Gaia}

\author{
J. PORTELL \\ Universitat Politècnica de Catalunya \\ Spain

\section{LURI} \\ University of Barcelona \\ E. GARCÍA-BERRO \\ Universitat Politècnica de Catalunya \\ Spain
}

Gaia is the most ambitious space astrometry mission currently envisaged and it will be a technological challenge in all its aspects. We describe a proposal for the payload data handling system of Gaia, as an example of a high-performance, real-time, concurrent, and pipelined data system. This proposal includes the front-end systems for the instrumentation, the data acquisition and management modules, the star data processing modules, and the payload data handling unit. We also review other payload and service module elements and we illustrate a data flux proposal.

Manuscript received December 19, 2003; revised November 30, 2004 and September 1, 2005; released for publication November $12,2005$.

\section{IEEE Log No. T-AES/42/2/876419.}

Refereeing of this contribution was handled by M. Ruggieri.

This work has been partially supported by the Generalitat de Catalunya (AGAUR) under FI Grant 2001FI 00715, and by the Spanish MCYT under Grant AYA2002-04094-C03-01. The Institute for Space Studies of Catalonia (IEEC), CIRIT, and DURSI (Generalitat de Catalunya) have also supported this work.

Authors' addresses: J. Portell, Institute for Space Studies of Catalonia and the Universitat Politècnica de Catalunya, Barcelona, Spain; X. Luri, Dept. of Astronomy and Meteorology, University of Barcelona and the Institute for Space Studies of Catalonia, Barcelona, Spain; E. García-Berro, Dept. of Applied Physics, Universitat Politècnica de Catalunya and the Institute for Space Studies of Catalonia, Barcelona, Spain, E-mail: (garcia@fa.upc.es).

0018-9251/06/\$17.00 (c) 2006 IEEE
ADCM Attitude data collection module

AF Astrometric field

AOCS Attitude and orbit control subsystem

ASM Astrometric sky mapper

ASTRO Astrometric Instrument

ATU ASTRO trail unit

BBP Broad band photometer

CCD Charge coupled device

CCSDS Consultative committee for space data systems

CDM Clock distribution module

DA Detection algorithm

DH\&C Data handling and compression

DSP Digital signal processor

FDI Field density index

FPA Focal plane assembly

HK Housekeeping

HKDCM Housekeeping data collection module

HRTF High rate telemetry formatter

IFDI Integrated FDI

mas Milliarcseconds

$\mu$ as Microarcseconds

MBP Medium band photometer

MTU MBP trail unit

OBS On-board storage

PDHS Payload data handling system

PDHU Payload data handling unit

PLM Payload module

PSF Point spread function

RON Readout noise

RTU RVS trail unit

RVS Radial velocity spectrometer

SA Selection algorithm

SDCM Science data collection module

SDPM Star data processing module

SDS Science data selection

SPECTRO Spectrometric and photometric instrument

SPF $\quad$ Source priority flag

SPV Supervisor module

SVM Service module

TDI Time delayed integration

TT\&C Telemetry, tracking and command

VC Video chain

VPU Video processing unit

WAM Window acquisition manager

WFST Wide field star tracker.

\section{INTRODUCTION}

Gaia is an ambitious space observatory, adopted within the scientific programme of the European Space Agency (ESA) in October 2000 [1]. It aims to measure the positions and proper motions of an extremely large number of stars and astronomical 
objects with unprecedented accuracy. As a result, a three-dimensional map of more than 1 billion stars of our Galaxy will be obtained, as well as solar system objects and extragalactic sources. The precision of the angular measurements will be about $10 \mu$ as at a moderate brightness (15th magnitude). In order to achieve this, Gaia will use two telescopes combined onto a single focal plane composed of 180 state-of-the-art charge coupled devices (CCDs). The satellite will continuously scan the sky, allowing for about 100 transits of each star during the 5 years of duration of the mission, each transit being composed of 16 measurements. Full sky coverage will be possible because of the spin of the satellite around its own axis, which itself precesses at a fixed angle of $50^{\circ}$ with respect to the Sun. A third telescope projects the sources on another CCD focal plane and will obtain spectra and medium band photometry of them, besides the angular (astrometric) measurements. This will lead to the most complete and accurate map of the stars of our Galaxy.

Gaia is the successor of Hipparcos, the first astrometric satellite, operated also by ESA from 1989 to 1993 [2]. Hipparcos was a very successful space mission, and some of its operating principles have been taken into account for Gaia. Other space missions similar to Gaia (such as OBSS [3], SIM [4], and JASMINE [5]) have been proposed, but are still under consideration. Some others (like DIVA [6] and FAME [7]) were also proposed but have been discarded.

Continuous measurement of stellar sources using CCDs implies a special operation, different than the typical shutter-based imaging. Time delayed integration (TDI) is the best option for this case, which is based on a continuous charge shift from one pixel row to the next, synchronized with the satellite spin motion. It will be done in each CCD, thus accumulating the charge during its corresponding integration period. In this way, long exposure times can be achieved without distortion or blur. The total transit time of a stellar source will be about $57 \mathrm{~s}$ in the astrometric focal plane, during which 11 measurements of $3.3 \mathrm{~s}$ each will be performed, among others. The total transit time in the spectrometric and photometric focal planes is about $145 \mathrm{~s}$.

Measuring more than 1 billion objects several times with the highest resolution implies a technological challenge, not only for the predicted 20 TB data base of compressed raw data (on ground), but also-and specially-for the on board data handling. Reading the whole set of CCDs entirely would imply a video output of more than $7 \mathrm{Gbit} / \mathrm{s}$ (only in the astrometric focal plane), so a selective sampling method is mandatory. This will be done by detecting and selecting the most interesting sources to measure, and then reading only windows (sets of pixels) around the detected sources. The sampling method is fully described in [8] and is beyond the scope of the work presented here. However, let us mention that for a typical case of single faint stars, the astrometric instrument will bin 12 pixels in the across-scan direction for obtaining a sample, that is, a single flux value. Afterwards six contiguous samples are read, in the along-scan direction, thus offering a pattern or shape of the point spread function (PSF) of the star.

Using this sampling method and the baseline technical features, the video data output rate of the astrometric focal plane may be reduced to less than $400 \mathrm{Mbit} / \mathrm{s}$ in the worst of the cases, namely, when a very dense stellar field is observed. Typical stellar fields should imply an output of about $200 \mathrm{Mbit} / \mathrm{s}$. Although this value may still appear large, typical communication standards like Spacewire can implement this payload data bus comfortably.

With a predicted launch in 2010, Gaia will orbit around the L2 Lagrangian point, 1.5 million $\mathrm{km}$ from the Earth opposite to the Sun. Therefore, the satellite will be seen from the Perth ground station for about $8 \mathrm{~h}$ per day. This, presumably, will be the only radio station used for receiving the data, so a permanent link between Gaia and the ground station will not be available. This implies that the $4 \mathrm{Mbit} / \mathrm{s}$ data link will be reduced to an effective downlink of about 1.2 Mbit/s. Therefore, all the data acquired by the astronomical instruments must be compressed (with lossless methods whenever possible), packetized, and stored on a mass storage system, waiting for being transmitted to ground during the next contact. More details about Gaia can be found in [9].

In this paper we elaborate on the payload data handling system (PDHS) of Gaia, from the observation instruments to the mass storage. We intend to propose a complete system design, not limiting ourselves to a list of scientific requirements for the PDHS, in order to offer a much richer input to the industrial teams that will be in charge of manufacturing the system. On the other hand, we also intend to offer to the interested reader an example of such a high-performance system. The main elements proposed here are the front-end modules of the astrometric instrument (ASTRO), the payload data handling unit (PDHU), and the data exchanges between all these modules. Some suggestions about a supervisor module (SPV) coordinating the PDHU and the instruments are also given. All these modules and submodules, as well as their input and output signals, are intended to be consistent with the simulators and designs currently available for Gaia. Overall, the main interest of this proposal is the need of being consistent with the intrinsic operation of Gaia. It implies the use of a high-performance data handling system, operating in real time and capable of being implemented as a pipeline, since in average about 600 sources per 
second will enter the astrometric focal plane and will be measured during about $1 \mathrm{~min}$. Furthermore, the actual number of stars entering the focal plane will vary in a large scale, from very few stars per second up to about 4000 stars per second, depending on the area of the sky surveyed.

The latest design of Gaia will be taken into account, as well as different sampling options depending on the brightness or kind of sources [8]. However, the option of selectable gate phases will be maintained, in order to allow for a reduction of the integration time for a given source. It is important to realize that along this paper we intend to offer a high-level description of the payload modules and data fluxes. Although some items may be described in detail, it is done just in order to avoid confusions. We also describe some third-party elements and designs such as the CCDs, for offering a better understanding of the overall operation of the payload.

The paper is organized as follows. In Section II an overview of the Gaia payload is given. In Section III the ASTRO is described, while the PDHU is described in Section IV. Section V lists the other elements in the service module of Gaia. Finally, in Section VI our conclusions are summarized.

\section{OVERVIEW OF THE PAYLOAD OF GAIA}

\section{A. Summary of Modules and Submodules}

The design of the payload of Gaia was elaborated by EADS-Astrium in 2002 under a contract with ESTEC. The payload contains two main instruments, named ASTRO and SPECTRO (spectrometric and photometric instrument). We focus on the former, which is the astrometric instrument. This instrument consists of two telescopes (ASTRO-1 and ASTRO-2), the images of which are combined and projected onto a single focal plane assembly (FPA), with 180 CCDs and the corresponding proximity electronics. A mask is applied to these images, in such a way that some of the CCDs are only illuminated either by one or another telescope, while the rest of CCDs receive the combined images. In this way, the system can determine the projecting telescope for every object. Fig. 1 helps in understanding this. In this figure we can see 10 CCD trails each following the apparent motion of the stars on the focal plane (along-scan direction), and $18 \mathrm{CCD}$ strips in the perpendicular direction (across-scan). This nomenclature [10] is used instead of the usual row and column nomenclature for avoiding confusions: within industrial environments these names are transposed from the usual meaning in a figure. The area illuminated by each of the telescopes is indicated by the dotted or dashed lines. The first CCD strip is illuminated only by

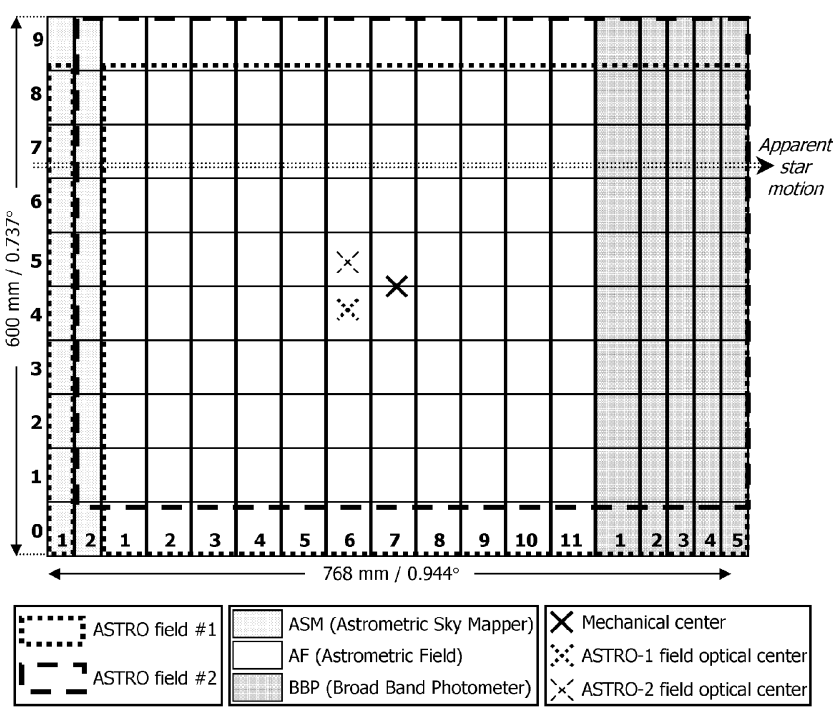

Fig. 1. Layout of astrometric focal plane corresponding to ASTRO instrument.

the ASTRO-1 telescope, while the second one is illuminated only by the ASTRO-2. The rest of the focal plane will contain combined images from both telescopes.

A CCD trail is a physical part of the FPA, containing both the CCDs and the proximity electronics needed for basic star acquisition. An ASTRO trail unit (ATU) is defined as a functional unit, including a set of video processing modules, which converts a CCD trail in a self-operated module. Each ATU is intended to operate independently, which leads to an intrinsic parallelism in the design and implementation of the proximity electronics and data handling. One of the options for the design could be to distribute this focal plane in 10 video processing units (VPUs), each linked to a trail of 18 CCDs and their proximity electronics. We propose a different distribution based on ATUs, which is more intuitive, efficient and easy to implement. Each one of these 10 ATUs contains the following elements.

1) One CCD trail, composed of 18 CCD chips, 18 video chains (VCs) and 18 local sequencers. The first two CCDs, named astrometric sky mappers (ASMs), will be in charge of detecting the sources entering the focal plane. They will also classify them, whether they are being observed by ASTRO-1 or ASTRO-2 telescope, thanks to the masks explained before. The next $11 \mathrm{CCDs}$, or astrometric field (AF), will perform the high-quality astrometric measurement, while the last five CCDs will perform broad band photometry (BBP).

2) One window acquisition manager (WAM), which will make possible the measurement of only the interesting areas of the sky.

3) Two star data processing modules (SDPMs), implementing the detection algorithm (DA) and the selection algorithm (SA), see Section IIIB below. The 


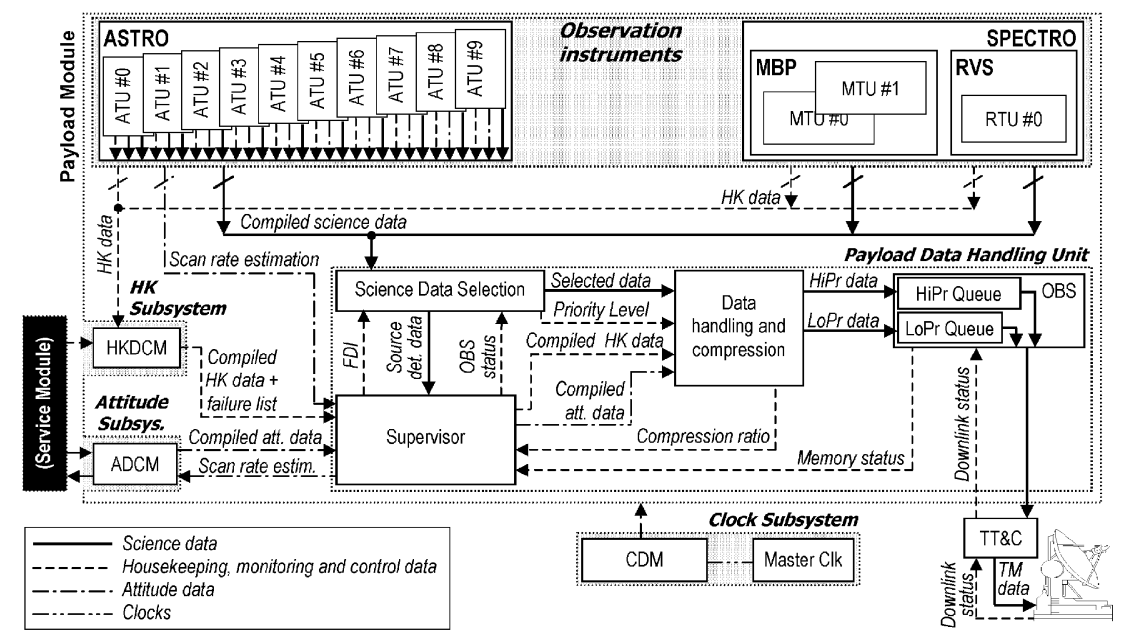

Fig. 2. Global view of Gaia payload, including main data interfaces and internal modules of PDHU.

DA will detect and confirm stellar sources entering the focal plane, also discarding false detections due to cosmic rays. The SA will select the sources to be measured and the quality of this measurement. Also, the SA will compute the real spin rate of the satellite using the measured star data, and will assign a priority flag to each source depending on its scientific interest.

4) A star data collection module (SDCM), compiling the data from all the CCD chips and the star data processing modules.

The SPECTRO instrument is composed of a single telescope. The center of its field of view will be projected over a spectrometer designed to measure radial velocities through Doppler shifts, hence its name, radial velocity spectrometer (RVS). A medium band photometer (MBP) will measure the outer parts of the field of the telescope. The technical implementation of SPECTRO in CCDs, VCs, sequencers, and other video data processing modules will be similar to the ASTRO implementation. It will also contain several functional units operating in parallel, named RVS trail units (RTUs) and MBP trail units (MTUs). Fig. 2 illustrates only 2 MTUs and 1 single RTU, which is the design envisaged for those instruments.

The PDHU, linked to both the instruments and the high rate telemetry formatter (HRTF), will be composed of the following elements.

1) Supervisor (SPV), which will control the overall operation of the instruments and data handling.

2) Science data selection (SDS), which will be in charge of selecting the data to be transmitted to ground taking into account storage and downlink limitations. Also an eventual lossy codification will be determined by this element, although all the data generated by Gaia should be transmitted with no losses whenever possible. Finally, the transmission priority will also be assigned by the SDS.
3) Data handling and compression (DH\&C), compressing and packeting the data according to Consultative Committee for Space Data Systems (CCSDS) standards.

4) On-board storage (OBS), also known as mass storage, which may be implemented as a solid-state recorder.

The attitude subsystem will contain an attitude data collection module (ADCM), compiling data from the attitude and orbit control system (AOCS), the wide field star tracker (WFST), and several gyroscopes. It is important to note here that, because the CCDs offer a high precision, the scan rate will be measured from the sky mappers and plays a major role in maintaining the required attitude accuracy. The housekeeping (HK) subsystem is composed of an HK data collection module (HKDCM) and several sensors placed both in the payload module (PLM) and in the service module (SVM). The clock subsystem is composed of a master clock, a clock distribution module (CDM), and several local counters distributed among the instruments and modules.

\section{B. Global Scheme and Data Flux}

Fig. 2 shows a global view of the payload of Gaia, including the main data fluxes between them. Some of the signals have been intentionally omitted or simplified for the sake of clarity, whereas some links to the SVM have been included for illustrative purposes. This figure clearly shows how we can consider the ASTRO instrument as 10 independent subinstruments, which we name ATUs. This independency is considered a requirement, and taking advantage of this parallelism when implementing the system in digital signal processors (DSPs) or microprocessors is strongly recommended. It is envisaged that the MBP and RVS instruments will operate with a similar parallelism, although these instruments are still under design. 


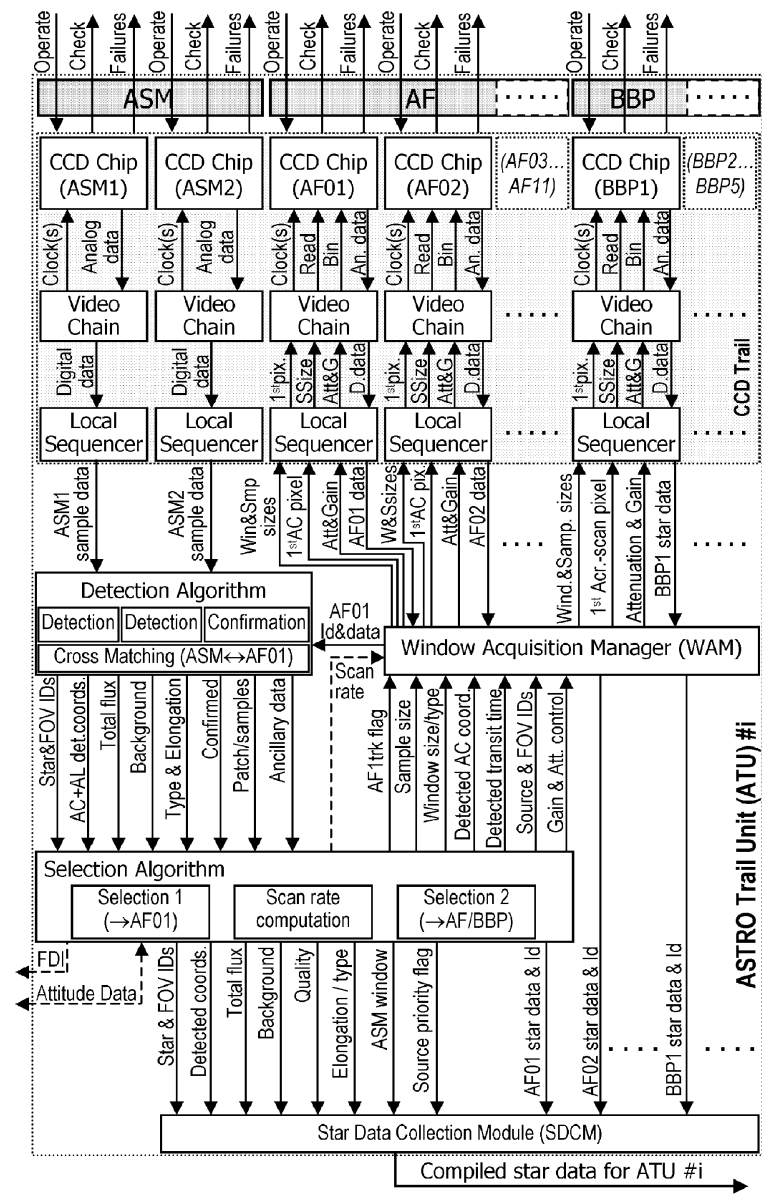

Fig. 3. Modules of data flux within ATU. Some interfaces, as well as a part of the CCD trail, have been omitted for sake of clarity. ASTRO is composed of 10 of these units, operating independently and in parallel.

\section{ASTROMETRIC INSTRUMENT (ASTRO)}

In this section the main interfaces, submodules, and operation overview of the ASTRO instrument of Gaia are described. We focus on the astrometric focal plane, which is the main operative element of the ASTRO instrument. A field identifier (ASM, $\mathrm{AF}$ or $\mathrm{BBP})$ is used for referencing its several parts, followed by a CCD strip number: $1 \ldots 2$ for the ASM, $01 \ldots 11$ for the AF and $1 \ldots 5$ for the BBP. The MBP and RVS instruments, still under design, will be based on a similar scheme. All these instruments can be considered as self-controlled systems, measuring the sources and sending the data to the appropriate module or submodule as they enter the focal plane. A few parameters are controlled by the SPV, as seen in the interface list below. Some inputs to the ASTRO instrument may not feed directly into the focal plane or into its proximity electronics, but may be filtered by the star data processing modules. A diagram of this system is given in Fig. 3. The following are the main inputs to ASTRO.
1) Attitude data from the ADCM, filtered by the SPV, needed for the positioning of the acquisition windows.

2) Operate signals from the SPV indicate if a given module or submodule is being operated or deactivated. It may be needed due to hardware problems, or during the transfer phase to the final orbit around L2.

3) Clock signals from the CDM must offer the adequate time resolution with the highest possible precision.

There will also be other inputs, which will be fixed most of the time, for opening the way to eventual in-orbit reprogramming. For instance, the SPV offers the maximum simultaneous samples value to every $\mathrm{CCD}$. This parameter indicates the maximum number of samples that can be read out at a given time from a CCD chip. This value affects the resulting read out noise (RON), because the higher this value, the faster the CCD will have to read the data. Actually, this value indicates the number of samples that are always (at any TDI period) read from the CCD. It is required for maintaining constant the power dissipation. Useless samples will be discarded.

The outputs of ASTRO may also be from other submodules, rather than from the focal plane itself, as follows.

1) Compiled source data, towards the SDS module, is the result of the observations made in this instrument.

2) $H K$ data, towards the HKDCM, contains a set of global checks and a failure list.

3) Scan rate estimate, towards the ADCM, offers an estimate of the scan rate. This output is filtered by the SPV before reaching the ADCM.

4) Field density index (FDI), towards the SPV, is an estimate of the star density of the field currently being observed. It will be useful for data selection and other issues [11].

These interfaces are applicable to each one of the ATUs. As previously mentioned, each one of these units contains a CCD trail, with 18 CCD chips aligned in the along-scan direction. Each CCD has its corresponding $\mathrm{VC}$ and local sequencer for commanding the image acquisition at a higher level. Fig. 3 illustrates this distribution, together with the internal and external CCD trail interfaces. The remaining video processing and management modules of an ATU are also illustrated there.

\section{A. Focal Plane and Proximity Electronics}

We define a CCD trail as a set of 18 CCD chips in the along-scan direction, with their corresponding VCs and local sequencers. The full set of 10 CCD trails conform the focal plane and proximity electronics. This concept can be easily identified as a physical 


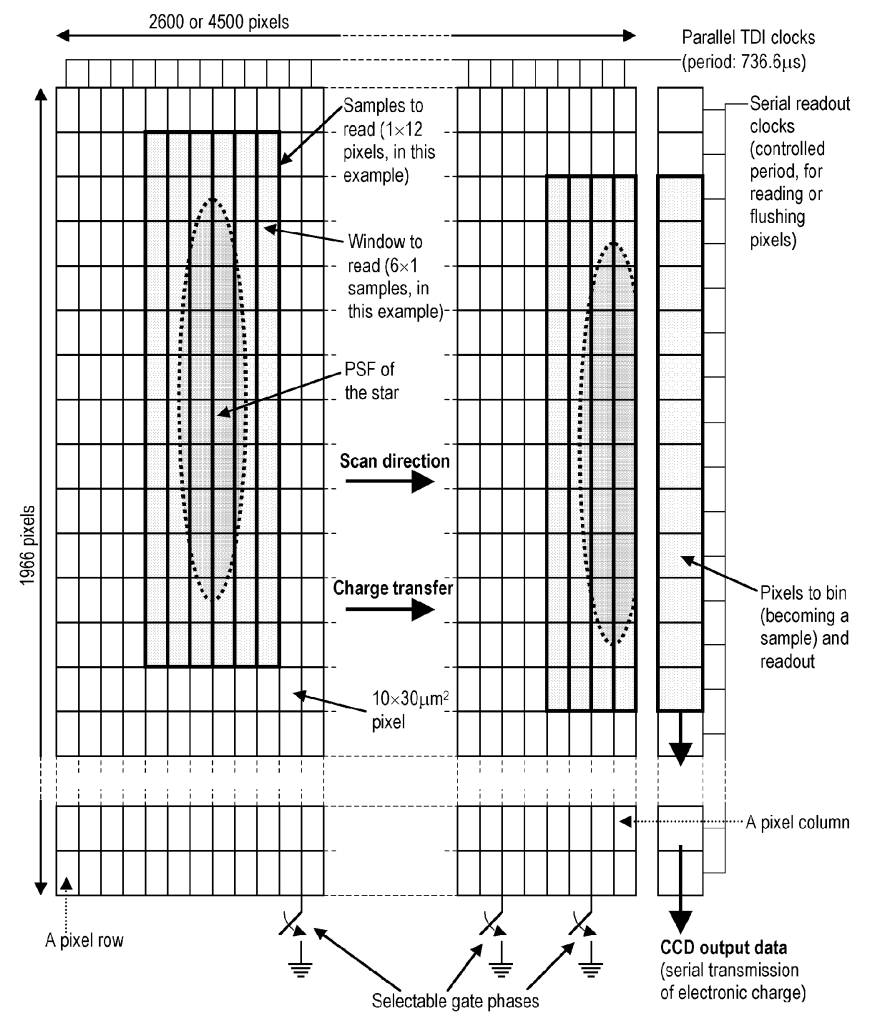

Fig. 4. Operation principle of CCDs to be used in astrometric focal plane (adapted from [12]). In average, several hundreds of stars will be projected on a CCD during its real operation, although only two stars are shown here.

element: the CCD focal plane with the electronics attached to it. On the other hand, an ATU is identified as an operative and standalone element. Here we describe the contents of a CCD trail, while the rest of elements within an ATU (the video processing and management modules) are described in Section IIIB below.

1) CCD Chips: The commanding method and interfaces of a CCD chip are highly industrial dependent, so the features described here will be rather general and based on the requirements listed in [12]. Their final implementation will be done under industrial contracts with ESA, but they must always operate in TDI mode and follow the main guidelines illustrated in Fig. 4. The transfer of charges in the CCDs must be perfectly synchronized with the apparent motion of the star images on the focal plane. In this way, the PSF of the image of a star will enter a CCD trail and will be tracked all along it, from CCD to CCD. Furthermore, several sources will be tracked simultaneously, both across-scan and along-scan. In the current design, up to 32 sources may be tracked simultaneously, across-scan, in AF02-10. The along-scan quantity of sources will depend on the stellar field, which ranges from a maximum of $3 \cdot 10^{6}$ stars $/ \mathrm{deg}^{2}$ to a typical value of $2.5 \cdot 10^{4} \mathrm{stars} / \mathrm{deg}^{2}$. These stellar field densities imply a typical rate of 267 stars/s per telescope, up to a maximum of 32000 stars/s.
The ASTRO contains narrow and wide CCDs, with 2600 or 4500 pixel rows, respectively. All of them have 1966 pixel columns. According to [10], we name row a pixel line in the across-scan direction, and column a pixel line in the along-scan direction. The active surface of the CCD can be considered as an array of parallel shift registers using the same TDI clock, as illustrated in Fig. 4. This figure shows also the read out register, a row of pixels with a higher capacity that is not illuminated. It can be considered as a serial shift register, outputting the pixels or samples one by one. The clock frequency used for this operation will depend on the kind of pixels being read. In this way, useless pixels will be flushed at high speeds, while useful pixels or samples will be read out more slowly. This selective read out method lowers significantly the resulting RON of the measurements, typically below $10 \mathrm{e}^{-}$. Therefore the useful dynamic range is improved, since the maximum capacity of a pixel well is above $200.000 \mathrm{e}^{-}$. The VC linked to each CCD is the only element commanding it, except for the operate signal which comes from the SPV, as follows.

1) Operate is an "on/off" binary switch for deactivating a CCD if it fails.

2) Read is a serial input indicating whether the pixel being shifted out from the read out register has to be read or flushed.

3) Bin is another serial input indicating whether the pixels being read must be binned (summed) or not. This input only has sense if read is active. A notch in this signal will cause a reset in the accumulator, thus making possible the read out of two consecutive samples. Another possibility could be to include a reset_sum_register input.

4) Gates, a multiple input, controls each one of the 12 selectable gate phases of the CCD. It activates them or not depending on when a pixel row has to be reset or not. Thus, bright stars may be measured this way, using a shorter integration time in order to avoid pixel saturation.

5) Clocks include a parallel clock, at about $1.36 \mathrm{kHz}$, distributed to all the pixel rows in order to perform the parallel charge shift. Also a serial clock, up to a few $\mathrm{MHz}$, is used in the read out register for serial pixel read out or flush. This read out clock will be different for flushed and useful pixels. Its valid values must accomplish a set of conditions for making possible the read out and flush of the entire read out register during a single TDI period.

The main output of a CCD chip is the analog data, which sends the accumulated flux of each pixel towards the corresponding VC. Also, the HKDCM is reported with the global check and list of failures of the CCD chip.

2) Video Chains: A CCD chip is the most elementary module that measures the data, treating its 


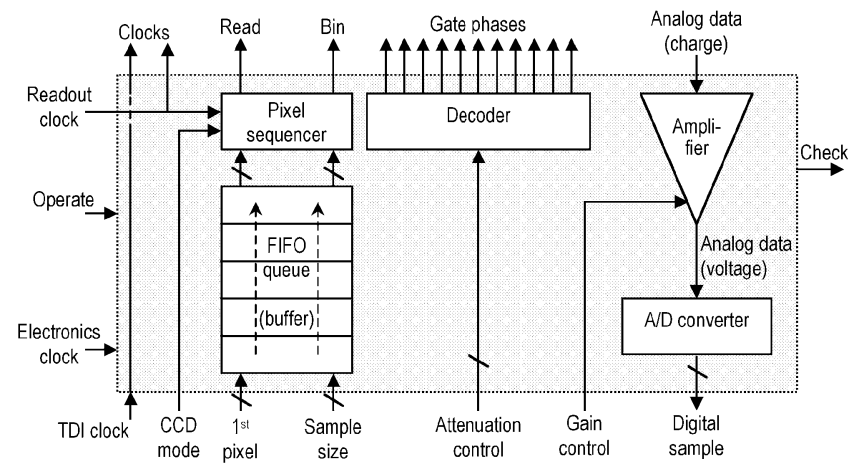

Fig. 5. Internal layout of VC. It operates as interface between CCD chip and local sequencer, translating mid-level command sets to low-level serial commands using corresponding TDI and read out clocks.

analog pixels one by one. A VC (or video controller) must be included for dealing with more useable data. It pre-processes the data, converting it from electronic charge to electronic voltages, and afterwards converting it to digital 16-bit values. Also, this module converts mid-level commands to low-level commands for the CCD. Therefore, every CCD chip must have its own VC, so an ATU will also contain 18 VCs.

A VC manages commands related to a single pixel row. For this reason a strict instruction protocol must be defined, which will be based on the TDI period of $736.6 \mu \mathrm{s}$ in the ASTRO. During this time, all of the pixels in the read out register of the CCD must be read or flushed. We define the transit time as the time when a given set of pixels of a star is loaded in the read out register. In other words, the PSF of a star image will be over the read out register at its transit time, although the read out register is actually not illuminated. With this in mind we define the commanding protocol for measuring a set of samples from a given pixel row. We assume here that their transit time is at TDI cycle $n$.

1) At the TDI cycle $n-1$ the $\mathrm{VC}$ receives a sequence of commands indicating the samples to be read.

2) During the TDI cycle $n$ the commands are executed. This will imply an output from the CCD chip. These analog data will be amplified, digitized, and sent to the local sequencer.

3) At the end of the TDI cycle $n$ plus the A/D conversion time, all the digital data will be available in the local sequencer.

This commanding protocol is further explained in Section IIIB1 below. Fig. 5 shows a possible implementation of a $\mathrm{VC}$, also including the gate phases and variable gain control. The FIFO queue stores the commands until their correct release time. Its size should be about 2 times the number of simultaneous samples that can be read out in a CCD. In this way, the commands for the current and the next pixel row can be stored.
This figure also shows the required inputs and outputs of a VC. Interfaces in the top of the figure link with the CCD chip, and they have been already described in Section IIIA1. Operate and check have the same meaning as in the CCD chip. An easier read out control for the SPV, as well as a higher flexibility in case of failure, is provided by other inputs. CCD mode is an example of this, which will mostly be fixed during the entire mission because of the pre-fixed behaviour of the several parts of the focal plane. It could be changed if necessary, thus forcing a windowed mode of a continuous mode. A CCD operating in windowed mode only reads pixels around each star. It is the typical mode for the AF and BBP. On the contrary, the continuous mode implies reading the whole $\mathrm{CCD}$, thus ignoring some of the VC inputs. It is the typical mode for the ASM.

The other inputs, all of them sent by the local sequencer, are required for commanding the windowed read out mode. 1st pixel indicates the first pixel (across-scan) to be read for a given sample, following the convention of pixel numbering as described in [10]. Sample size indicates the number of across-scan pixels to be read and binned for the current sample. Attenuation control indicates the gate phases to activate for reducing the integration time, while gain control selects a low $\left(3 \mu V / \mathrm{e}^{-}\right)$or high $\left(6 \mu \mathrm{V} / \mathrm{e}^{-}\right)$amplification for the $\mathrm{VC}$ amplifier.

The data read out from the CCD chip and converted to a digital value is finally sent in 16-bit format, offering a high enough resolution. A logarithmic-like A/D converter offering higher resolutions at lower signal levels could be an interesting alternative, and may be studied in the future.

3) Local Sequencers: VCs operate only with data measured during one TDI cycle, acquiring them from the read out register of the CCD. On the other hand, local sequencers are able to manage two-dimensional data, acquired during several TDI cycles. Therefore, they deal with patches and windows, which are sets of samples both along and across scan, while VCs deal only with samples. The use and basic requirements of both VCs and local sequencers were already introduced in [12], but we propose here a detailed operation for these modules.

A CCD local sequencer receives read out commands related to a given star with a fixed anticipation with respect to its transit time. In this way, unnecessary signaling and electronics near the focal plane are avoided, such as the transmission of source identification. Two TDI cycles of anticipation are recommended, thus leaving 1 cycle for sequencing tasks and another one for VC tasks. Combining several source read commands into sample read command sequences is the main task of a sequencer, which will release these commands towards the VC at 


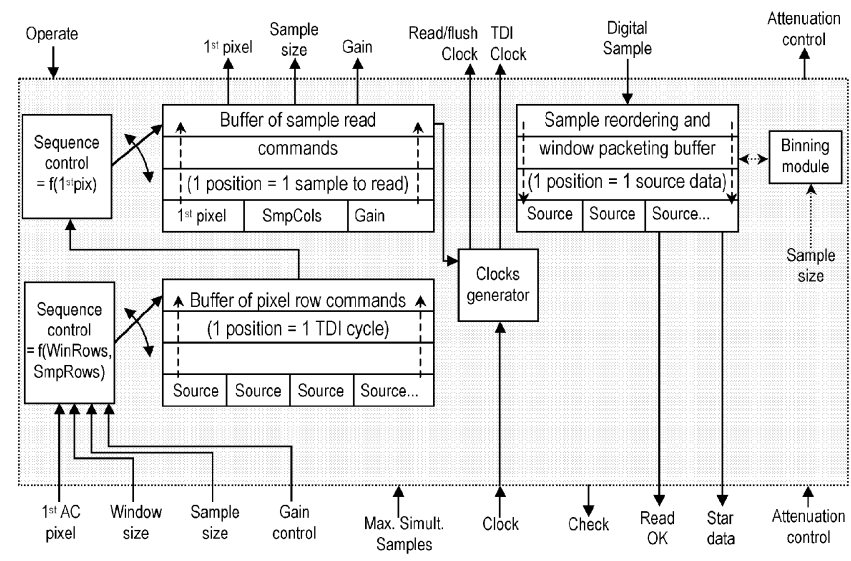

Fig. 6. Possible implementation of CCD local sequencer. We recommend implementing it on a DSP or similar. It is the main external interface of focal plane, receiving high-level commands and returning data blocks with all samples from each star.

their adequate time. We will come back to this issue when discussing Fig. 8 in Section IIIB1.

A local sequencer is always linked to a $\mathrm{VC}$ and its corresponding CCD chip. It can be considered as the main external interface of the focal plane. The WAM controls the local sequencers in order to obtain the measurements during the passage of a given star along the focal plane. As explained before, the WAM will send commands to the sequencers 2 TDI cycles before the transit time of each star. These commands include, as shown in Fig. 6, the window and sample sizes to be used for a given star. As a requirement, all the samples within a window must have the same size. Also, only commands for contiguous windows will be accepted. This implies that some measurements, mainly those of bright stars, will have to be requested as two different measurements, both for the same star.

The 1st AC pixel is another required input for acquiring star data. It indicates the first across-scan pixel of the window to be acquired, which corresponds to the uppermost pixel to be read as seen in Fig. 1. It follows both conventions of pixel numbering described in [10]. Max simult. samples, introduced in Section III, is an example of those inputs required for an eventual in-orbit reprogramming. It tells the sequencer the number of samples that must be read in the CCD chip during a TDI cycle. If the requested samples are not enough for reading this amount of samples, the sequencer will complete this number requesting dummy samples. It is important for maintaining constant the power consumption in the focal plane. Fluctuations around the nominal value may lead to thermal variations, which must be avoided due to the mission requirements. About 32 to 64 samples must be continuously read in most of the CCDs in the current design.

Star data is the main output of a local sequencer. The local sequencer sends to the WAM the set of samples acquired for a given stellar object. Also, a Read OK flag indicates if this source has been successfully read. If not, only a partial observation (PO) will be available for this star. A reason for this may be a sample overlapping, or a window falling out of the CCD bounds. It must be noted that samples from different stars cannot overlap on the CCDs, although windows can do it if necessary [8].

Fig. 6 illustrates these and other interfaces. Interfaces in the top of the layout have been already described in Section IIIA2 before, as well as check and attenuation control. A single clock input will be required at the master clock frequency, namely 6.4 MHz. Other clocks for TDI and read out operation will be generated by using, e.g., frequency dividers.

The acquisition requests for a local sequencer will be partitioned in sample commands and fed into a queue of pixel row commands. These will be sorted depending on several issues, such as the window width or the sample width. Sample commands for several sources will be combined in this buffer. All the commands related to a star must fit in this queue, so its size must be larger or equal to the widest of the windows that can be acquired. According to [13] the number of registers must be at least 68 . One of these registers will be served every TDI cycle, feeding them into another buffer. This second queue will contain as many registers as simultaneous samples to be acquired in the CCD. Each register will contain sample requests commands, ordered using the 1st $\mathrm{AC}$ pixel value. These commands will be served at the beginning of a new TDI cycle, being sent to the $\mathrm{VC}$ at their corresponding time, see Fig. 8 in Section IIIB1 below.

Digital samples received from the VC will be recombined for each source. This recombination is possible because a fixed commanding protocol will be used, and because sample data will be received from the VC during the TDI cycle next to its request. Also, useless samples, for maintaining a constant read out rate, will be discarded during the recombination process. Finally, remaining binning operations will be performed, and sample sets from each star will be combined in order to offer star data blocks. Ultimately, all these data will be sent to the WAM at the beginning of the next TDI cycle.

\section{B. Video Processing and Management Modules}

The focal plane and proximity electronics are composed of $10 \mathrm{CCD}$ trails, which will be able to acquire star data as requested. Our proposal for the modules in charge of selecting the stars to be acquired, command the CCD trail, and process the results are described in the next subsections.

1) Window Acquisition and Management: The aim of this module is to accurately control the several sequencers of the CCD trail for acquiring the star 


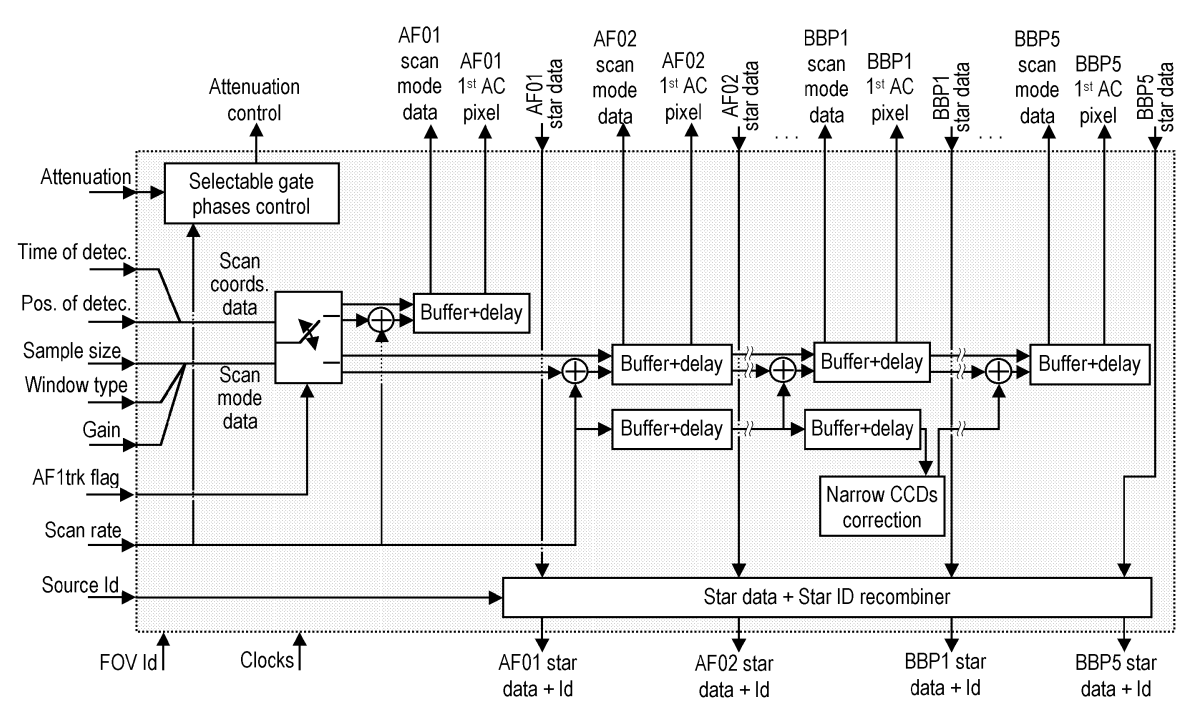

Fig. 7. Internal layout of a WAM. Controls for AF03 to AF11 and BBP2 to BBP4 have been omitted for sake of clarity.

data, and to forward these data towards the PDHU. It is commanded mainly by the SA, which sends the basic coordinates for each star to be tracked. From these, the WAM obtains the sequence of transit times and transmits the individual acquisition coordinates to each local sequencer. We note here that the CCDs of AF01 measure the data in a different way than the rest of the CCDs of the AF, being its main task the confirmation of the source. Because of this, the SA inputs a special AF1trk flag into the WAM. With a value of true, it indicates that the detection (ASM) coordinates are input, and that AF01 confirmation coordinates must be computed. Otherwise, it indicates that confirmation (AF01) coordinates are input, and that AF02-11 and BBP measurement coordinates must be computed. This is illustrated in Fig. 7, where AF1trk $=$ false propagates the acquisition coordinates all along the CCD trail.

Fig. 7 also illustrates the several interfaces of the WAM. One of these is the scan rate, which is an input from the attitude subsystem. The scan rate is a vector indicating the apparent motion of the sources on the focal plane. Also the coordinates (time and across-scan position) where the source has been detected or confirmed are provided. The WAM will take these origin coordinates and add the scan rate to them several times. Each time this addition is performed, the acquisition coordinates for a new CCD strip will be obtained. These transit predictions will always be calculated taking into account the attitude at the detection or the confirmation time, not at the time when the star is measured in the AF or in the BBP. We note the narrow CCDs correction element in the layout, which corrects the calculation of the transit times for being adequate to CCDs BBP2-5, which are narrower than the rest of CCDs.

The sampling method, which includes the sample size and window type, is also provided by the SA, as well as the gain and attenuation values. These parameters will be different for AF1-10, AF11, and BBP fields, so multiple inputs are required. Also a Source Id input is necessary for uniquely identifying the source being measured and, thus, making possible the compilation of its data at the end of the measurements. This identifier can be as simple as a counter, with a recycling time large enough. We note that the WAM outputs each CCD measurement one by one, while the SDCM will compile all of them (see Section IIIB4 below). Neither this identifier nor the transit times will be transmitted to the local sequencers, thus avoiding unnecessary interfaces. The acquisition protocol shown in Fig. 8 makes it feasible. The results of the star measurement are sent to the WAM exactly win_width +3 TDI cycles after requesting the measurement, taking win_width as the width of the acquisition window in pixels. Data from source measurements with the same win_width and requested during the same cycle will be processed on a first requested-first output basis. Sources projected by one or another telescope (ASTRO-1 or ASTRO-2) are detected by ASM1 or ASM2, respectively. It implies different calculations for the transit times. Therefore, a field id flag must also be served.

2) Detection Algorithm: The way in which Gaia measures the stars has already been explained previously. However, we still have to show how Gaia knows what (and where) to measure. The DA is the key for this.

The CCDs of the ASM are the first ones where the images of the stars are projected. These CCDs are read as a whole, and the result is a continuous image strip, a kind of panoramic picture of the sky. The DA receives this strip and looks for sources in it. Point-like stars are the most typical sources, but there can also be binary stars, galaxies, near-Earth objects (NEOs), solar system bodies... all of them with 


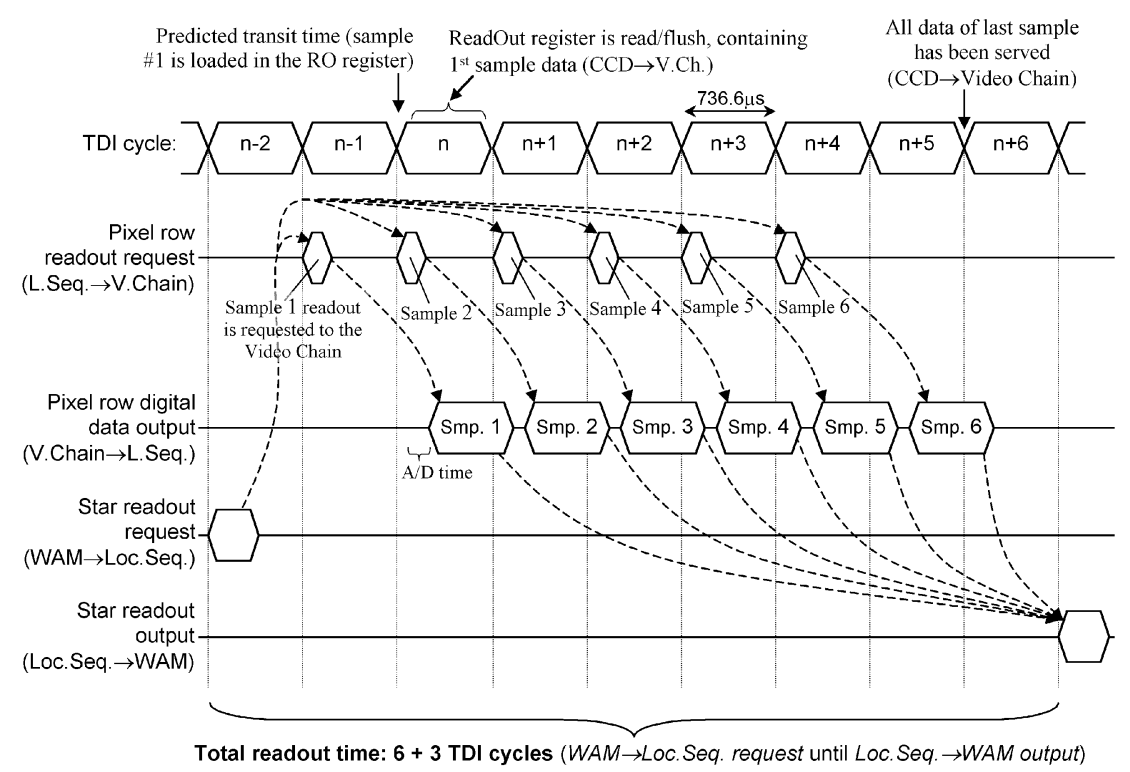

Fig. 8. Timing protocol for acquisition of star data. Typical sampling ( 6 samples of 12 pixels each) is assumed. Readout process will operate as a pipeline, so the 3 extra TDI cycles are not strictly dead times.

a wide range of brightness, up to magnitude 21 . Every source will also have different background conditions, motions, and shapes. Overall it makes the design of an image DA a very complex task [14].

The DA should be implemented in a set of microprocessors or DSPs, taking advantage of the intrinsic parallelism of Gaia (see Section IIB). It is the video processing module which has the most extreme requirements, both in timing restrictions and processing load. Therefore, it is imperative to implement the DA as a concurrent and pipelined set of parallel processes, whichever physical implementation is used. Its processing capability must also be correctly sized, so that very dense stellar fields can also be analyzed.

From the operational point of view, the DA is composed of four subprocesses. Two of them are identical, although operating with different inputs-ASM1 and ASM2. They receive the continuous image strips, and looks for sources in these images. Not only the coordinates and features of the detected sources will be obtained, but also a background estimate will be computed and a detection quality flag will be issued. A third subprocess is in charge of confirming the detections, using AF01 measurements. Although these images are not in a continuous strip form but as windows around the detections, the operation of this subprocess is also similar to the previous ones. The main difference is that the background estimate is taken from the preliminary detections, and that a better image quality is used, because only a fraction of the CCD is read. Finally, a fourth subprocess is in charge of cross-matching the detections and the confirmations. It will not only verify that detection occurs both in ASM (first detection) and in AF01 (confirmation), but it will also verify the main features of the source. In this way, false detections due to cosmic rays or read out noise will be minimized.

The main outputs of this module will include the coordinates of the detection with an adequate resolution. A pixel-based resolution is recommended: $1 / 250$ of pixel will be enough for avoiding important quantization errors. Also the validity and quality of the detection will be an output, as well as the source features. It includes the elongation, the type of acquisition windows to use, and the total flux measured, as well as a unique identifier. The field (ASM1 or ASM2) where it has been detected must also be an output, thus indicating if the source is projected by the ASTRO-1 or the ASTRO-2 telescope. Finally a set of samples surrounding the ASM detection, as well as the AF01 window, will be sent for completing the measurement data block.

3) Selection Algorithm: Once the several sources entering the focal plane have been detected and confirmed, Gaia must select the most interesting of them. The current status and capability of the PDHS, as well as the detection features, must be taken into account. The most interesting sources will always be measured, while the remaining sources will be measured depending on the current system load and on the stellar field density. Selecting the sources to measure (and the way it has to be done) is the aim of the SA. It will depend on the available resources. Data selection, in order to decide what to transmit, even what to store on-board, will be done in a second stage. This will be performed by the SDS module in the PDHU (see Section IVA below).

Acquisition modes for every confirmed source will be selected by the SA. It includes the gain, attenuation, and sampling mode. Also a small shift 
of the acquisition window may be selected, which will be notified to the WAM outputting bogus detection coordinates. A reason for doing this could be the presence of a nearby source.

One of the main features of the SA is the capability of calculating a source priority flag. Each source will be classified in this way, numerically indicating its scientific interest. Key factors for its calculation include the multiplicity or shape of the source, its brightness and the quality of its detection. This flag will be useful for both selection stages in Gaia, so that measurement, storage, and transmission of a source will be an easier decision. The source priority flag (SPF) is calculated during the first SA stage, when processing ASM detection data. Taking AF01 hardware limitations, first-detected sources will be selected for trying to confirm them in AF01 or not. It is named Selection 1 in [8] and in Fig. 3. Confirmed sources will correct their SPF value, because of the higher quality of AF01 measurements.

The SA will continuously compute another useful value for PDHS purposes. It is the FDI, an indicator of the density of the stellar field currently being scanned. This value, as well as the SPF, will be used for on-board control purposes. Therefore none of them will be included in the science telemetry at most in the HK telemetry. A long-term index, the integrated FDI (IFDI), will also be calculated by integrating the FDI evolution during a few seconds. Both values, FDI and IFDI, will be computed taking into account all the confirmed sources, even those not selected for being measured.

ASM detection and AF01 confirmation are separated in time by 3.6 or $5.8 \mathrm{~s}$, respectively, depending on whether the sources are projected onto the ASM1 or ASM2. This value and the resolution of the measurements are high enough for obtaining a good estimate of the scan rate. This is a third task to be done by the SA, which will make possible an accurate tracking of the sources on the focal plane. Moreover, the attitude determination will be obtained with a higher resolution than in the AOCS. The next stage of the SA is to determine the samples and acquisition windows to be used when measuring the source in the rest of the CCDs (AF02-11 and BBP). This stage includes the overlapping test, checking if a nearby source would overlap its window with the window of the current source. If this is the case, the algorithm will try to accommodate the windows by shifting their centers. If this is not possible, one of the sources will be discarded. Another possibility is that the windows overlap only in some CCDs. It can be possible with one source from every telescope, because the apparent across-scan motion will be opposite. In this case, a PO may be selected [8]. The last stage, selecting the most interesting sources to be measured all along the focal plane (AF02 until BBP5),

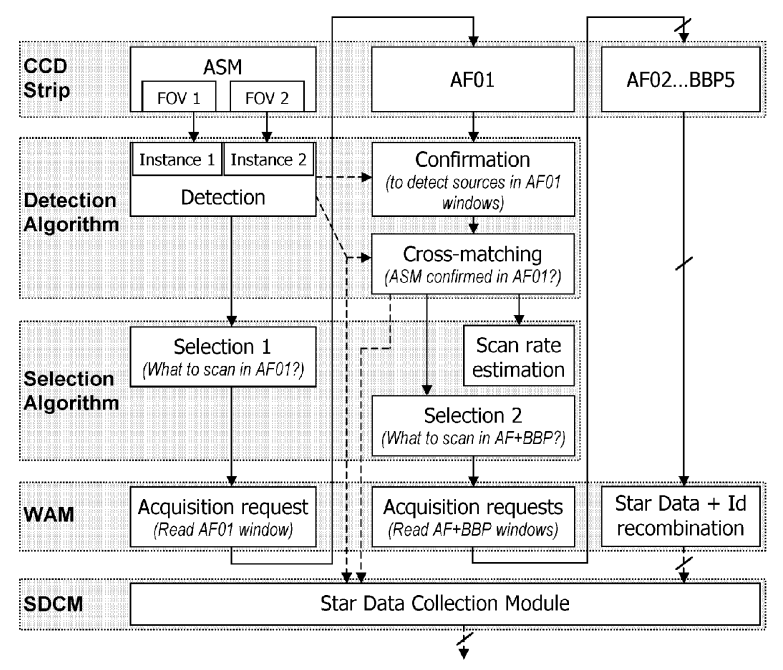

Fig. 9. Operational diagram of ASTRO. Solid lines indicate flux diagram, dashed lines give a hint on the data path. It includes samples from ASM detection, AF01 confirmation and measurement, and AF02-11 and BBP measurements.

is named Selection 2 in [8] and in Fig. 3. All these measurements will be requested to the WAM.

4) Science Data Collection Module: This module simply collects all the detection and measurement data of a source, sending it afterwards to the PDHU. These data are inputs from the SA and the WAM, including not only the data to be transmitted to ground but also possible extra flags. An example is the source priority flag, to be used when assigning the transmission priority to the data. A flag indicating the ATU (0 to 9) is also included by the SDCM itself. The cross-matching of all the data related to the same source is possible thanks to the source id, embedded in the SA and WAM data.

\section{ASTRO Operational Diagram}

The diagram in Fig. 9 shows the process of detection, confirmation, selection, and measurement of a star entering the astrometric focal plane. Some names of the operations are taken from [8]. In this figure the three main tasks in the measurement of a source are displayed as three columns: detection, selection, and high-quality measurement.

\section{PAYLOAD DATA HANDLING UNIT}

Perhaps, one of the most critical issues of Gaia is transmitting the huge amount of data generated by the instruments and, hence, it is one of the main challenges in its design. Measuring only a set of small images around each source already decreases this amount of data by a large factor. However, the preselected data may still not fit in the downlink. The PDHU, as we propose here, must decide which of these data must be retained for other contacts, and which must be directly discarded. 
The PDHU should be implemented in some kind of programmable device, preferably a general-purpose microprocessor because of the varied tasks to perform. After the DA it will be the module with the highest processing requirements. Several microprocessors will probably be used operating in parallel, taking into account the limited processing power of space-qualified devices. The details of the tasks to perform may change after Gaia has been launched or even during the lifetime of the mission. For example, an optimized data compression scheme may be developed when real measurements will be eventually available. Therefore, the devices implementing the PDHU should be reprogrammable in orbit. This is the reason why we recommend that some data fluxes go through this module, e.g., the scan rate or the HK data. Overall it will provide a higher flexibility, even in case of failure. Several processes will operate concurrently for implementing the PDHU. We describe each of them below.

\section{A. Science Data Selection}

The three selection criteria operating in Gaia are fully described in this section. Source selection depends on the hardware resources, and it is performed by the SA in the instruments. The other two are applied by the SDS. The first one takes into account the amount of memory available on-board, and decides whether to store or discard the source data. The second one takes into account the downlink capability, in order to delete the data or transmit it with a given priority.

An input from the OBS (Section IVD below) informs about the memory available in each of the multi-priority queues. With this information, together with the field density indexes, a resource availability index is computed. This index gives a hint on how restrictive should the selection be. An estimate of the data compression ratio will also be taken into account, provided by the DH\&C module (Section IVC below). Science data is afterwards indexed in a stack depending on the source priority flags. This stack is then emptied by blocks, from higher to lower priority, sending the science data towards the DH\&C. Lowest priority data may even be discarded if compression resources are not enough. In the compression module different methods will be applied to the data depending on their priority, but specially on the resource availability. If memory or telemetry resources are very low, lossy compression methods may be used for the low priority data. These methods may be as simple as discarding some of the high-resolution measurements, or even transmitting only detection data. The losses, however, must be directly controlled by the data system. It means that compression systems such as wavelets or JPEG will not be applicable at all.

\section{B. Supervisor}

This is the process in charge of controlling most of the PDHS modules and subprocesses, as well as the data flux between them. Failures are also controlled here. If one CCD chip, VC, or local sequencer reports a failure, the supervisor will turn it off. A substitute for this will be selected in the case that it is required. For example, an ASM failure would be fatal for the operation of the focal plane. Then, an AF01 would operate as ASM, and AF02 as AF01 [8].

Attitude data will be filtered by the SPV before offering a final scan rate estimate. It will select the most well-behaved sources such as single bright stars. In this way, sources with high proper motions will not be included in the estimate, thus approaching closely the actual attitude of the satellite. This selection may also be applied to the FDI index if necessary. IFDI is also calculated here, which offers a long-term indication of the stellar density being measured. This integrated index will be a key factor in the SDS module: combined with the resource availability index it will indicate if the selection restrictions can be relaxed or not. The control of the evolution of these indexes makes it possible. For example, the resources may be low at a given moment, and the density indexes be high. If the tendency is decreasing, the SDS can safely assume that the generation of science data will decrease, and so it can assign more resources to it.

\section{Data Handling and Compression}

It is another crucial process in the data path of Gaia, since there is still too much data to downlink, even taking into account the sampling method and the selection of sources and data. Studies like [15] indicate that between 5 and $7 \mathrm{Mbit} / \mathrm{s}$ per second of raw data will be generated. This implies that a data compression ratio between 4 and 6 must be obtained. Only in this way can we fit these data in the $1.2 \mathrm{Mbit} / \mathrm{s}$ per second available in the downlink. Furthermore, a lossless compression system is imperative, because no data from a source measurement can be lost. At most, its resolution or measurement multiplicity could be reduced. The data to be transmitted are not raw images or data with a clear and uniform structure, so standard compression systems do not satisfy the requirements. There are some essays on data compression for Gaia $[16,17]$ which offer interesting results, such as ratios higher than 3, although they are simple systems. The idea of partitioning the science data in uniform blocks, and to use differential coding for repeated measurement data, seems to be the right approach. It implies that the DH\&C must have a data partitioner offering subframes with uniform data. Afterwards, a data analyzer will look for redundancy 
patterns in each block of data, selecting the most appropriate data compressor for it. This process may be suppressed and the data redirected with a fixed scheme, depending on the final compression system. A set of independent data compressors, such as Huffman, LZW, or Rice, will properly compress the set of data subframes. All of them must operate in parallel. The real compression ratio will be measured afterwards, and reported to the SPV and SDS. Finally, the telemetry frames will be generated. They will include the compressed science data, as well as the HK and attitude data. CCSDS standards are met in the packet and frame generation.

\section{On-Board Storage}

Selected and processed data must be temporarily stored on-board until a downlink contact is established. A solid-state mass memory recorder will surely be used for this, implementing some kind of file system for differencing the priority levels. One file will be created for every priority level, although Fig. 2 represents only 2 levels as data queues. The priority is labeled on each block of data, thanks to the SDS. When entering the OBS it will store a data block in one or another queue, i.e., file. The HRTF directly links with the OBS. When downlink availability is reported data queues are emptied, from the highest to the lowest priority. Low priority data that cannot be transmitted during that contact will be stored if possible, waiting for the next contact (and slightly increasing its priority level), within a limited range. We note here that the OBS cannot reorder or manage its data. It only inputs, stores, and outputs data sequentially, although there are several concurrent processes for the different priority levels. We can compare the OBS with a set of FIFO queues.

\section{OTHER MODULES}

Most of the modules listed here may be standard industrial components, ready to be included in the Gaia payload or service module. Only an overview of their required operation and interfaces is included here for the sake of completeness.

\section{A. Attitude Data Collection Module}

This module receives attitude data from both the ASTRO instrument and the AOCS. The latter offers gyroscopes and WFST measurements, while the former gives a higher resolution provided by the CCDs. The task of the ADCM is to compile all of these data and redirect them towards the appropriate module. It can be considered as the interface between the payload and service module for attitude data.

\section{B. Housekeeping Data Collection Module}

All the HK data reported by the payload systems converge into this module, as well as those from several sensors placed in the service module. The HKDCM compiles and monitors them in order to report errors to the SPV. One of the most important sensors is the basic angle monitor. It is a laser device offering an accurate measurement of the angle between the two astrometric fields of view. Its knowledge is crucial for obtaining a final astrometric map with the highest precision. Therefore, this and other HK data will be selected by this module for being included in the telemetry frame.

\section{Clock Distribution Module}

The main purpose of this module is to distribute the several timing products to the payload elements. It is a critical module, because the quality of the final products of the mission depends highly on its correct design. The reason is the high resolution of the measurements, which require a precision in the datation of the order of $10 \mathrm{~ns}$. The main timing product is the master clock, implemented as a rubidium atomic clock offering a frequency of about $6.4 \mathrm{MHz}$. This product must be distributed uniformly, i.e., avoiding phase differences between elements of the instrumentation. Also a real-time clock must be obtained, which can be as simple as a counter of nanoseconds since a given reference time or reset. Other clocks, such as the TDI or read out clocks, will be obtained in the local sequencers or other PDHS elements. These subproducts must be obtained with the highest precision achievable.

\section{SUMMARY}

The main operational principles of the Gaia mission have been introduced here, together with an overview of its technical implementation. We have focused on its payload, and more specifically on its data handling. The main instrument, the astrometric focal plane, has been thoroughly described. An implementation option for the data handling has been proposed, following the whole data path step by step. Beginning from the simplest data structures to the most complex ones, the focal plane and proximity electronics have been explained. It contains the CCD chips which just read pixels and offer samples. These samples are amplified and digitized by the $\mathrm{VC}$, and the local sequencers compile a whole measurement of a star. Beyond the proximity electronics, the WAM compiles all the measurements and detection data for outputting the data block of the star passage. The video processing modules include not only the WAM, but also the DAs and SAs. The former detects the stars entering the focal plane, and the latter selects 
which will be measured depending on its detected features. The focal plane and proximity electronics, together with the video processing modules, conform each one of the 10 ATUs.

We have recommended assigning a priority level to each block of data received by the PDHU, which will determine the data to be transmitted during the next contact. Data with lower priority will only be transmitted if there is enough downlink capability. These data are analyzed, coded, and compressed, storing the result into one or another queue depending on its priority. The several high-capacity queues will be emptied in order during the next contact. A supervisor controls all this process and the instruments operation. Other modules in the payload and service module have also been reviewed, such as the clocks, attitude, and HK subsystems.

This proposal of PDHS takes into account all of the requirements of the Gaia mission. These are really restrictive, so that a fully optimized data system was necessary. Here we accomplish this objective, offering a real-time capable system. Furthermore, parallelism is present in many subsystems, while others are proposed as pipelines. All of this eases the final implementation and makes possible the management of such a huge amount of data generated by the instruments.

\section{ACKNOWLEDGMENT}

We acknowledge F. Arenou for very valuable discussions and criticisms, for a careful reading of the original manuscript and for suggesting to us to undertake the study of the PDHS of Gaia. F. Safa has gently reviewed the main technical aspects and confidentiality issues of this paper. S. Mignot, C. Babusiaux, and I. Mart'n are also acknowledged for their very useful comments and corrections. We also thank the whole Gaia team of Barcelona for their support and enlightening discussions.

\section{REFERENCES}

[1] Perryman, M. A. C., de Boer, K. S., Gilmore, G., Hoeg, E., Lattanzi, M. G., Lindegren, L., Luri, X., Mignard, Pace, F. O., and de Zeeuw, P. T.

GAIA: Composition, formation and evolution of the Galaxy.

Astronomy \& Astrophysics, 369 (2001), 339.
[2] ESA

The Hipparcos and Tycho Catalogues.

1997, ESA SP-1200.

[3] http://ad.usno.navy.mil/OBSS/

[4] http://planetquest.jpl.nasa.gov/Navigator/sim_library.html.

[5] http://www.jasmine-galaxy.org/.

[6] http://www.ari.uni-heidelberg.de/diva/.

[7] http://www.usno.navy.mil/FAME/.

[8] Høg, E., Arenou, F., Mignot, S., Babusiaux, C., Katz, D., and Jordi, C.

Scientific requirements for the on-board processing. GAIA-CUO-117, 2003, http://gaia.am.ub.es/PWG/ documents/GAIA_CUO_117.pdf. See also http://gaia.am.ub.es/PWG/documents.html for more information.

[9] http://sci.esa.int/gaia/.

[10] Portell, J., Garc'a-Berro, E., Luri, X., and Jordi, C. Proposal of reference systems for Gaia. GAIA-BCN-002, version 2.0, 2002, http://gaia.am.ub.es/Portell/GAIA-BCN-002.pdf.

[11] Portell, J.

Some ideas for GIBIS and beyond. GAIA-BCN-003, 2001, http://gaia.am.ub.es/Portell/GAIA-BCN-003.pdf.

[12] ESA

Gaia concept and technology study report, ESA-SCI(2000)4, 2000.

[13] Høg, E.

Sampling in Astro of bright stars and double stars. GAIA-CUO-100.3, 2002, http://obswww.unige.ch/ eyer/VSWG/docs/A100.ps.

[14] Chéreau, F.

Gaia_Detect description.

OBD-FC-01, rev. 1.3, 2002, http://stellarium.free.fr/cv/OBD-FC-01.ps.

[15] Portell, J., Masana, E., Garc'a-Berro, E., and Luri, X. Proposal of telemetry formatting for Gaia science data. GAIA-BCN-005, version 0.2, 2002, http://gaia.am.ub.es/Portell/GAIA-BCN-005.pdf.

[16] Portell, J., Garc'a-Berro, E., and Luri, X. Flux data codification: Proposals of simple codification schemes.

GAIA-BCN-001, version 2.0, 2001, http://gaia.am.ub.es/Portell/GAIA-BCN-001.pdf.

[17] Portell, J., et al.

Payload data handling, compression and telemetry formatting.

Presented at the GAIA Simulation Working Group kick-off meeting, Cambridge, UK, Mar. 12, 2002, http://gaia.am.ub.es/Portell/PDHU-CompressionPrintable.pdf. 

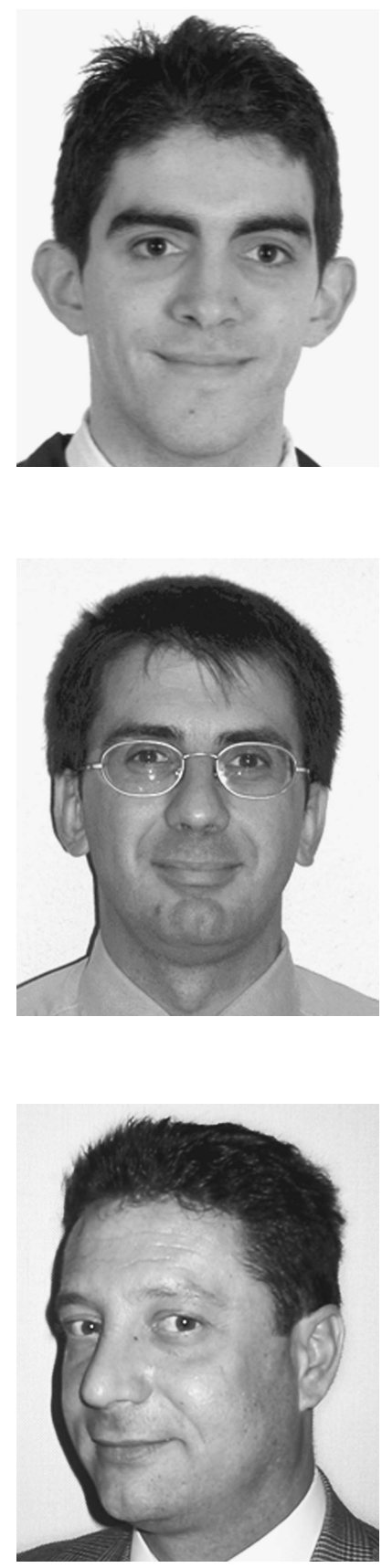

Jordi Portell received his degree in electronic engineering from the Universitat Politècnica de Catalunya (UPC), Barcelona, Spain, in 2000. In 2002 he received the M.S. degree in applied physics from the same university.

$\mathrm{He}$ is currently pursuing a Ph.D. in applied physics at UPC, in collaboration with the Institute for Space Studies of Catalonia (IEEC), Barcelona. His research activities are focused in Gaia, including the modeling of the astrometric instrument, reference systems, and payload data handling. Apart from this, his main interest is the telemetry and data compression of the satellite. He has also worked on the preliminary design of a Spanish minisatellite, SIXE. Since then, he has actively collaborated in the definition of several technical documents for being circulated in the scientific and technical teams of both missions.

Xavier Luri graduated in physics at the University of Barcelona (UB) in 1989. He earned his Ph.D. in astrophysics at the same university in 1995. Since 2003 he is lecturer in the Astronomy Department of UB, and research associate of the Institute for Space Studies of Catalonia (IEEC) since 2000.

His research interests span from purely astrophysical subjects related to the study of our Galaxy, to the development of mathematical methods applied to astronomical problems, or to technological fields related to the design and development of scientific satellites and payloads. He is presently member of the Gaia Science Team, in charge of the scientific coordination of the design and construction of Gaia. He is also coordinator of the Simulation Working Group of Gaia and member of the Spanish Astronomical Society.

Enrique García-Berro earned his Ph.D. in astrophysics from the University of Barcelona in 1987.

He was postdoctoral research associate at the University of Illinois at Urbana-Champaign. He is now professor at the Department of Applied Physics at the Universitat Politècnica de Catalunya (UPC) since 1991. He is also research associate of the Institute for Space Studies of Catalonia (IEEC) since 1996. His research interests have a broad scope ranging from theoretical astrophysics to the design and implementation of scientific payloads for astronomical satellites.

Dr. García-Berro has published more than 50 papers in refereed journals. $\mathrm{He}$ is a member of the Spanish Astronomical Society and of the American Astronomical Society. 